\section{Correlação do pH e volume salivares com sintomas laringofaríngeos}

\author{
Henrique Olival Costa', \\ Cláudia Alessandra Eckley²
}

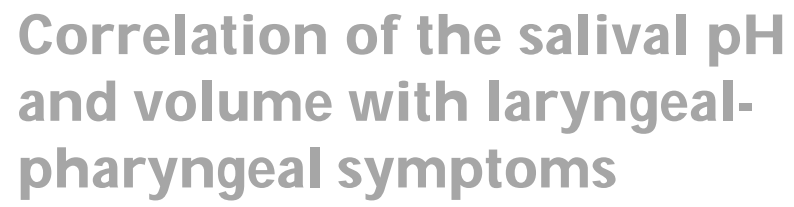

Palavras-chave: laringite, saliva, diagnóstico, $\mathrm{pH}$, volume. Key words: laryngitis, saliva, diagnosis, $\mathrm{pH}$, volume.

Resumo / Summary $\mathrm{n}$ spite of the great enthusiasm wakened up by the progress of the concepts of the denominated syndrome of the larygopharyngeous reflux (LFR), it is still difficult for the otorrinolaringologist to establish with safety if this illness can be considered in the future as a disease similar to the gastro esophageal reflux. The inflammatory signs in the segment, found in some patient that present phmetry with $\mathrm{pH}$ below 4 in the proximal probe can be found in other situations aggressive to the area, not being, therefore, patognomonic of LFR. The conditions of saliva, the volume, clearence and alterations of the eletrolitic conditions can influence the capacity of protection of the regional mucous membrane. Aim: To observe the relationship between the inflammatory symptoms and the $\mathrm{pH}$ and volume of saliva. Study Design: Observacional cohort with transversal cut. Material and Method: 59 subjects were studied, with ages varying from 24 to 76 years, with average 50,5 years, being 44 women and 15 men. All the patients answered to a questionnaire on laryngopharyngeous symptoms and they had his/her saliva collected and measured its volume and $\mathrm{pH}$. Results: The median volume of saliva collected in the patients was $4,3 \mathrm{ml}$, with the minimum of 1,5 and a maximum of 7,5 . The median $\mathrm{pH}$ was of 7,1, extending from 6 to 8 . Of the total of symptoms 31 patients presented dysphonia, 39 throat clearing, 2 dyspnea, 24 halitosis, 4 caseum, 4 recurrent tonsillitis, 6 teeth and gingival problems, 9 oral ulcers, 6 xerostomia, 12 glossodynia, 36 globus pharingeus, 2 odinophagia and 8 dysphagia. The several correlations among $\mathrm{pH}$, volume to salivate and symptoms were observed showing, in some cases, strong positive or negative correlation. Conclusion: The pH of saliva, in the dependence of its volume can have strong interference in the laryngopharyngeous inflammatory symptoms. ou negativa. Conclusão: 0 pH salivar, na dependência do volume salivar, pode ter forte interferência na sintomatologia faringolaríngea.

\footnotetext{
${ }^{1}$ O torrinolaringologista, Cirurgião de Cabeça e Pescoço, Prof. Adjunto da Santa Casa de São Paulo. 2 Otorrinolaringologista, Prof. Assistente da Santa Casa de São Paulo.

Endereço para Correspondência: Rua Prof. Artur Ramos, 183 cj.34 São Paulo SP 05414-000.

Artigo recebido em 14 de agosto de 2003. Artigo aceito em 15 de janeiro de 2004.
} 


\section{INTRODUÇÃO}

Apesar do grande entusiasmo despertado pelo avanço dos conceitos definidores do refluxo laringofaríngeo (RLF), ainda é muito difícil para o otorrinolaringologista estabelecer com segurança se esta síndrome poderá ser considerada no futuro como uma doença semelhante ao refluxo gastroesofágico ${ }^{1}$. A nosso ver, os sinais inflamatórios no segmento laringofaríngeo encontrados em alguns pacientes que apresentam phmetria com presença de $\mathrm{pH}$ abaixo de $5 \mathrm{na}$ sonda proximal podem ser mimificados por outras situações que agridem a região, não sendo, portanto, patognomônicos de RLF. Dentre as possibilidades de agressão local estão a hiperacidez causada seja por inalação de cigarro, a ingestão de alimentos cáusticos ou em extremos de temperatura e as infecções gengivais, nasais ou orofaríngeas ${ }^{2-6}$. Além destas eventualidades, as condições de fluxo salivar, seu volume, clearence e alterações das condições eletrolíticas da saliva podem influenciar na capacidade de proteção da mucosa regional ${ }^{7-12}$

0 equilíbrio entre os fatores de agressão e de proteção faringolaríngeos tem sido estudado em seus diversos aspectos, seja na mensuração de fatores epiteliais como expressão de proteínas (TNF, EGF e p53), seja nas substâncias que possam contribuir para a agressão tecidual como a pepsina, 0 ácido clorídrico e a presença de fungos e bactérias $^{13-16}$.

Sabendo que a saliva é uma das principais responsáveis pela manutenção da homeostase bucal, contribuindo para a estabilidade do $\mathrm{pH}$ e flora orais, o conhecimento das relações entre $0 \mathrm{pH}$ salivar e seu volume com sintomas laringofaríngeos associados ao RFL passa a ser um fator de extrema importância na clínica, tanto para a terapêutica quanto para o diagnóstico das afecções inflamatórios deste segmento.

A té o momento, pouca atenção foi dirigida no sentido de reconhecer qual é a composição salivar ideal e quais as mudanças determinadas por fatores como nível de hidratação, alimentação, exercício e estado emocional. Este conhecimento seria importante se a saliva, de fato, exercer um papel na homeostase regional e poderia ser determinante na produção de sinais e sintomas locais. Para esclarecer isto, Chicharro et al. ${ }^{17}$ estudaram os efeitos da atividade física na composição salivar. Segundo os autores, o exercício poderia induzir mudanças em vários componentes salivares como imunoglobulinas, hormônios, lactato, proteínas e eletrólitos. Os autores consideram que a composição da saliva poderia ser usada como um indicador da resposta dos tecidos de corpo aos diferentes esforços físicos. Neste respeito, a resposta de amilase e eletrólitos salivares para incrementos de atividade física seria de interesse particular. Segundo seus estudos, além de uma certa intensidade de exercício, e coincidindo com o acúmulo de lactato no sangue, há uma nítida elevação dos níveis salivares de alfa-amilase e eletrólitos (especialmente $\mathrm{Na}+$ ).

Interessantemente, algumas drogas que foram bastante utilizadas no tratamento dos pacientes com sintomas laringofaríngeos ocasionados pelo refluxo gastroesofágico também podem provocar alterações na composição salivar. Dentre elas, a cisaprida, uma droga procinética, tem mostrado em alguns estudos a capacidade de aumentar o volume salivar e, conseqüentemente, a capacidade de ácido-proteção. Chen et al. ${ }^{18}$, estudaram, por intermédio da cintilografia, 55 pacientes com esofagite de refluxo. Dividiu sua amostra em dois grupos, em um grupo manteve os pacientes em jejum e ofereceu cisap rida e no segundo manteve os pacientes alimentados e também ofereceu cisaprida. 0 fluxo salivar foi estudado antes e depois da ingestão da droga. Houve aumento significativo do volume salivar nos sujeitos que receberam cisaprida após a alimentação.

Também os inibidores de bomba de prótons parecem ter efeitos sobre a produção de saliva. Foram investigados mecanismos de formação fluida primária através de glândulas mandibulares de cangurus vermelhos que usam íontransporte e inibidores de anidrase carbônica na secreção salivar. Após a ingestão de butesamida, os cangurus apresentaram, simultaneamente, decréscimo de $[\mathrm{Na}],[\mathrm{Cl}]$ e da osmolaridade, além de aumento da composição de [K] e [HCO3]. Já quando os animais receberam bumetanida associada a amilorida houve aumento de [K] e [HCO3] e diminuição de $[\mathrm{Cl}]$, sem qualquer efeito no [Na] ou no fluxo salivar total ${ }^{19}$.

Como vemos, há implicações diversas na capacidade de tamponamento e na possível ação protetora que a saliva exerce quando estamos submetidos a condições não totalmente estudadas. Estas condições são pouco conhecidas e poderiam se devidamente compreendidas e manipuladas alterar o curso de uma afecção tão prevalente como o dito Refluxo Laringofaríngeo.

\section{OBJETIVO}

Este estudo teve como principal objetivo observar a relação entre os sintomas laringofaríngeos de inflamação e o $\mathrm{pH}$ e volume salivares.

\section{MATERIAL E MÉTODO}

Foram estudados 59 sujeitos com idades variando de 24 a 76 anos, com média 50,5 anos, sendo 44 mulheres e 15 homens. Os pacientes foram selecionados a partir de uma amostra aleatória de sujeitos seguidos no ambulatório de Gastroenterologia do Hospital Central da Santa Casa de São Paulo e encaminhados para avaliação da presença ou ausência de refluxo laringofaríngeo. Todos os pacientes encaminhados entraram na amostra. Não houve critérios de exclusão, uma vez que não tínhamos uma hipótese nula a ser testada e o trabalho se propunha a fazer uma prospecção 
inicial da relação ph e volume salivar e sintomas de refluxo laringofaríngeo. Todos os trinta pacientes apresentavam sintomas de refluxo distal e, portanto, foram submetidos a phmetria esofágica, exames estes que puderam ser comparados aos resultados do $\mathrm{pH}$ e volume salivar. Os outros foram sorteados junto ao ambulatório de O torrinolaringologia e completaram o grupo, sendo observadas as características de sexo e idade constantes no grupo submetido a phmetria.

Todos os pacientes assinaram termo de consentimento livre a esclarecido ap rovado pelo Comitê de Ética da Santa Casa de São Paulo.

Todos os pacientes responderam a questionário baseado em trabalhos que correlacionam RLF e sintomatologia ${ }^{20}$ ${ }^{22}$, onde sintomas como dispnéia, disfonia, pigarro, tosse, odinofagia, globus, aftas, amigdalites recorrentes, glossodínia, problemas dento-gengivais, presença de caseum na orofaringe e disfonia foram observados.

Os pacientes foram solicitados a permanecerem em jejum por um mínimo 8 horas antes da coleta, com a média de 10,3 hs de jejum, sendo que foram orientados a não fazerem uso de dentifrício no dia da coleta do material. A coleta foi processada após os pacientes lavarem a boca com água corrente para eliminar descamação epitelial e restos bacterianos. Os pacientes foram colocados em posição sentada, sem deglutir e um recipiente de vidro foi colocado abaixo do lábio inferior. A saliva que escorreu espontaneamente da boca no período de 15 minutos e foi coletada através de um funil acoplado a um tubo de ensaio que estava acondicionado em uma caixa de isopor com gelo. Seu volume total foi mensurado e o $\mathrm{pH}$ do volume recolhido foi constatado através de fita de $\mathrm{pH}$.

O s resultados obtidos foram submetidos a teste estatístico de correlação para avaliar as diversas possibilidades de associação entre sintomas e pH e volume salivar.

\section{Análise estatística}

Os resultados de cada um dos parâmetros foi comparado através de análise de regressão simples para pares $X$ e $Y$ baseado em método de menor quadrado com o modelo:
$\mathrm{Y}=\mathrm{b} 0+\mathrm{b} 1 * \mathrm{X}+$ erro, para avaliar a correlação entre $\mathrm{opH}$ e o volume da saliva e os sinto mas apresentados. Também os achados da pH metria foram correlacionados com o pH salivar.

\section{RESULTADOS}

0 volume médio de saliva coletado no total de pacientes foi 4,3 ml, com o mínimo de 1,5 e um máximo de 7,5. 0 pH médio foi de 7,1, se estendendo de 6 a 8. Do total de sintomas apresentad os 31 pacientes apresentaram disfonia, 39 pigarro, 2 dispnéia, 24 halitose, 4 caseum, 4 amigdalite de repetição, 6 problemas dento-gengivais, 9 aftas, 6 xerostomia, 12 glossodínia, 36 globus pharingeus, 2 odinofagia, 16 tosse e 8 disfagia.

Os resultados das correlações (entre -1 e 1), estão expostos nas Tabelas 1 e 2.

Também foi pesquisada a média volumétrica da saliva de acordo com os níveis de $\mathrm{pH}$ e a média para o pH 6 foi de $3,2 \mathrm{ml}$, para pH 7 foi de $4,1 \mathrm{ml}$ e para $\mathrm{pH} 8$ foi de $4,3 \mathrm{ml}$.

Para averiguar se a sintomatologia poderia estar correlacionada ao volume de saliva de acordo com o nível de $\mathrm{pH}$ foi observada a presença de sintomas por $\mathrm{pH}$ versus volume salivar. Os resultados estão apresentados nas Tabelas 3 , 4 e 5.

\section{DISCUSSÃO}

Como podemos perceber pelos resultados obtidos, há correlação negativa entre o valor do pH geral e a presença de sinto matologia laringo faríngea nos pacientes analisados. Tínhamos a expectativa de que o volume salivar geral pudesse estar relacionado com o pH geral final da saliva o que não ocorreu. De fato, o volume salivar médio foi menor nos pacientes com pH menor (6) que nos pacientes com pH maior ( 7 e 8) (3,2 versus 4,1 e 4,3). Para testar a possibilidade de o volume salivar estar correlacionado com os sintomas de acordo com o nível do $\mathrm{pH}$, fizemos o teste de correlação entre os diversos sinto mas e o volume por grupo

Tabela 1. Resultado de análise de co-relação entre pH salivar e fatores epidemiológicos e sintomatológicos

\begin{tabular}{|c|c|c|c|c|c|c|c|c|c|c|c|c|c|c|c|}
\hline & Idade & Sexo & $\begin{array}{l}\text { Volume } \\
\text { Salivar }\end{array}$ & $\begin{array}{c}\text { Sintomatologia } \\
\text { Faringolaríngea } \\
\text { Geral }\end{array}$ & Halitose & $\begin{array}{l}\text { Pigarro } \\
\text { Tosse }\end{array}$ & Tosse & $\begin{array}{c}\text { Globus } \\
\text { pharyngeus }\end{array}$ & Afta & Disfonia & Xerostomia & Caseum & $\begin{array}{l}\text { Problemas } \\
\text { Dento- } \\
\text { Gengivais }\end{array}$ & $\begin{array}{c}\text { Amigdalite } \\
\text { de Repetição }\end{array}$ & Glossodinia \\
\hline$\overline{\text { pHsalivar }}$ & $-0,079$ & 0,283 & 0,1327 & $-0,4331$ & $-0,341$ & $-0,334$ & $-0,163$ & $-0,623$ & $-0,287$ & $-0,480$ & $-0,260$ & $-0,130$ & 0,128 & $-0,253$ & $-0,098$ \\
\hline
\end{tabular}

Tabela 2. Resultado de análise de co-relação entre pH salivar e fatores epidemiológicos e sintomatológicos

\begin{tabular}{|c|c|c|c|c|c|c|c|c|c|c|c|c|c|c|}
\hline & Idade & Sexo & $\begin{array}{c}\text { Sintomatologia } \\
\text { Faringolaríngea } \\
\text { Geral }\end{array}$ & Halitose & $\begin{array}{l}\text { Pigarro } \\
\text { Tosse }\end{array}$ & Tosse & $\begin{array}{c}\text { Globus } \\
\text { pharyngeus }\end{array}$ & Afta & Disfonia & Xerostomia & Caseum & $\begin{array}{l}\text { Problemas } \\
\text { Dento- } \\
\text { Gengivais }\end{array}$ & Amigdalite & $\begin{array}{l}\text { Glossodinia } \\
\text { de Repetição }\end{array}$ \\
\hline $\begin{array}{l}\text { Volume } \\
\text { salivar }\end{array}$ & 0,099 & 0,196 & 0,0694 & $-0,001$ & $-0,097$ & $-0,154$ & $-0,084$ & $-0,133$ & 0,009 & 0,014 & 0,025 & 0,112 & 0,040 & 0,112 \\
\hline
\end{tabular}

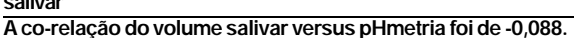


Tabela 3. Resultado de análise de co-relação entre volume salivar e sintomas, nos pacientes com pH salivar = 6

\begin{tabular}{lcccccccccc}
\hline Halitose & Pigarro & Tosse & $\begin{array}{c}\text { Globus } \\
\text { Pharyngeus }\end{array}$ & Afta & Disfonia & Xerostomia & $\begin{array}{c}\text { Caseum } \\
\text { Problemas } \\
\text { Dento- } \\
\text { Gengivais }\end{array}$ & $\begin{array}{c}\text { Amigdalite } \\
\text { de Repetição }\end{array}$ & $\begin{array}{c}\text { Glossodinia } \\
\text { Volume }\end{array}$ \\
Salivar & $-0,998$ & $-0,532$ & 0,576 & $-0,903$ & $-0,576$ & 0,620 & 0,576 & 0,000 & 0,000 & 0,576 \\
\hline
\end{tabular}

Tabela 4. Resultado de análise de co-relação entre volume salivar e sintomas, nos pacientes com pH salivar $=7$

\begin{tabular}{|c|c|c|c|c|c|c|c|c|c|c|c|}
\hline & Halitose & Pigarro & Tosse & $\begin{array}{c}\text { Globus } \\
\text { Pharyngeus }\end{array}$ & Afta & Disfonia & Xerostomia & Caseum & $\begin{array}{c}\text { Problemas } \\
\text { Dento- } \\
\text { Gengivais }\end{array}$ & $\begin{array}{c}\text { Amigdalite } \\
\text { de Repetição }\end{array}$ & Glossodinia \\
\hline $\begin{array}{l}\text { Volume } \\
\text { Salivar }\end{array}$ & 0,175 & $-0,029$ & $-0,431$ & $-0,001$ & 0,088 & 0,099 & $-0,008$ & 0,028 & $-0,130$ & $-0,130$ & 0,066 \\
\hline
\end{tabular}

Tabela 5. Resultado de análise de co-relação entre volume salivar e sintomas, nos pacientes com pH salivar = 8

\begin{tabular}{|c|c|c|c|c|c|c|c|c|c|c|c|}
\hline & Halitose & Pigarro & Tosse & $\begin{array}{c}\text { Globus } \\
\text { Pharyngeus }\end{array}$ & Afta & Disfonia & Xerostomia & Caseum & $\begin{array}{l}\text { Problemas } \\
\text { Dento- } \\
\text { Gengivais }\end{array}$ & $\begin{array}{l}\text { Amigdalite } \\
\text { de Repetição }\end{array}$ & Glossodinia \\
\hline $\begin{array}{l}\text { Volume } \\
\text { Salivar }\end{array}$ & $-0,176$ & $-0,359$ & 0,176 & 0,261 & $-0,314$ & 0,0 & 0,117 & 0,0 & 0,442 & 0,0 & 0,0 \\
\hline
\end{tabular}

de $\mathrm{pH}(6,7$ e 8$)$. Neste momento a correlação foi bastante nítida para alguns agrupamentos como nos casos em que $p$ $\mathrm{pH}$ salivar era baixo e a halitose $(-0,998)$ o que significa que não houve casos de halitose que não apresentasse volume salivar menor que o geral do grupo ou no caso de globus para o mesmo grupo $(-0,903)$. Para os grupos de $\mathrm{pH}$ neutro ou alcalino esta correlação foi menos nítida, mas ocorreu para $\mathrm{pH} 7$ e tosse (-0431) e para $\mathrm{pH} 8$ e problemas dentogengivais $(0,442)$, pigarro $(-0,359)$ e afta $(-0,314)$. Estes achados vão ao encontro da opinião de Cianci et al. que perguntam se o refluxo alcalino é fator de agressão. Nós temos esta impressão quando vemos sinais inflamatórios no riinofaringe de lactentes.

Este estudo tinha por objetivo fazer uma prospecção em um terreno ainda pouco explorado, mas que mostrava um rico potencial clínico em uma entidade nosológica de grande morbidade e prevalência.

Ao findar esta etapa podemos sugerir que o estudo deve ser ampliado e novas correlações devem ser estudadas como tipo e rotina dietética, tabagismo, condições gengivais e condições nasosinusais, que são outros fatores que podem se relacionar com o pH regional e interferir nos dados.

Contudo, as evidências de correlação das condições de tamponamento e clearance salivar com a sintomatologia laringofaríngea do chamado refluxo laringofaringeo mostram que podemos estar lidando com uma enfermidade de cunho local e que tratamentos objetivando alterar as condi- ções estomacais ou esofágicas podem estar sendo efetivas devido aos efeitos indiretos na boca e não necessariamente devido à alcalinização do estômago (anti-ácidos com bloqueadores $\mathrm{H} 1$ e inibidores de bomba de prótons) ${ }^{24,25}$. Estudos recentes revelam uma grande interferência na secreção de $\mathrm{K}, \mathrm{HCO} 3$ e Cl na saliva de roedores quando da ingestão de inibidores de bomba de prótons. Por outro lado, há evidências de que outras medicações utilizadas anteriormente para melhorar a cinética esôfago-estomacal, como a cisaprida, também faziam efeito no fluxo salivar, aumentando seu volume se tomados após as refeições.

A constatação de que o pH salivar se relaciona de alguma maneira com os sintomas laringofaríngeos, na dependência da quantidade de saliva produzida, assim como a constatação de que o pH mais alto ocorre em sujeitos com volume salivar maior, e ainda o fato do $\mathrm{pH}$ salivar não guardar uma grande correlação com a pHmetria $(0,164)$ nos faz pensar que temos uma nova possibilidade diagnóstica ambulatorial e não invasiva na mensuração do volume e pH salivares ${ }^{26}$. Também podemos começar a pensar em novas estratégias terapêuticas para minimizar os sinto mas da entidade denominada Refluxo Laringofaringeo.

\section{CONCLUSÃO}

$\mathrm{O}$ pH salivar, na dependência do volume salivar, pode ter forte interferência na sintomatologia faringolaríngea. 


\section{REFERÊNCIASBIBUOGRÁFICAS}

1. Gavazzoni FB, De Ataíde AL, Herrero Junior F, Macedo Filho ED. Esofagite por refluxo e laringite por refluxo: estágios clínicos diferentes da mesma doença? Rev Bras ORL 2000; 68(1): 86-90.

2. Adhami T, Goldblum J, Richter J, Vaezi M. Role of gastric and duodenal ingredients in laryngeal tissue injury: an experimental study in dogs. Abstracts of the Digestive Disease Week 2002; 429: A-87.

3. Da Wes C. Circadian rythms in human salivary flow rate and composition. J Physiol 1972, 220: 529-45.

4. Korsten MA, Rosman AS, Fishbein S, Shlein RD, Goldberg HE, Biener A. Chronic xerostomia increases esophageal acid exposure and in associated with esophageal injury. Am J Med 1991; 90: 701-6.

5. Maccini DM \& Veit BC. Salivary Epidermal Growth factor in Patients with and Without Acid Peptic Disease. Am J Gastroenterol 1990; 85(9): 1102-4.

6. Benamouzig R, Ferriere F, Guettier C, Amouroux J, Coste T, Rautureau J. Role of salivary and seric epidermal growth factor in pathogenisis of reflux esophagitis in chronic alcoholics and nondrinkers. Dig Dis \& Sci 1996; 41(8): 1595-9.

7. Moazzez R, Anggiansah A, Bartlett $D$, Owen W. Tooth wear saliva and symptoms of GERD: is there a relationship? Abstracts of the Digestive Disease Week 2002; WI164: A-816.

8. Christensen ME, Therkildsen MH, Poulsen SS, Bretlau $P$. Transforming growth factor alpha and epidermal growth factor in laryngeal carcinomas demonstrated by immunohistochemistry. Acta Oto-Laryngol 1993; 113(4): 563-7.

9. Namiot Z, Rourk RM, Piascik R, Hetzel DP, Sarosiek J, Mccallum RW. Interrelationship between Esophageal Challenge with Mechanical and Chemical Stimuli and Salivary Protective Mechanisms. Am J Gastroenterol 1994; 89(4): 581-7.

10. Dagogo-Jack S. Epidermal growth factor EGF in human saliva: effect of age sex race pregnancy and sialagogue. Scand J Gastroenterol 1986; 21 (Suppl 124): 47-54.

11. Nandurkar S, Cameron A, Fett S, Zinsmeister A, Locke III GR. Environmental causes of reflux: influence of lifestyle diet and psycholgical factors. Abstracts of the Digestive Disease Week 2002; S1356: A-268.

12. Gray MR, Donnelly RJ \& Kingsnorth AN. Role of salivary epidermal growth factor in the pathogenisis of Barrett's columnar lined oesophagus. Br J Surg 1991; 78: 1461-6.

13. Orsini B, Brocchi A, Calabrà A, Fedip, Tommasi MS, Surrenti C. Radioimmunoassay of Epidermal Growth Factor in Human Saliva and Gastric Juice. Clin Biochem 1991; 24: 135-41.
14. Gill GA, Arthur C, Hampson F, Dettmar P, Moorghen M, Pigna Telli M. Characterization of acid and pepsin damaged laryngeal and oesophageal mucosa. Abstracts of the Digestive Disease Week 2002; TII15: A-595.

15. Mcgurck M, Hanford L, Justice S, Metcalfe RA. The Secretory Characteristics of Epidermal Growth Factor in Human Saliva. J Oral Biol 1990; 35(8): 653-9.

16. Sarosiek J, Scheurich CJ, Marcinkiewicz M, Mccallum RW. Enhancement of Salivary Esophagoprotection: Rationale for a Physiological Approach to Gastroesophageal Reflux Disease. Am J Gastroenterol 1996; 110: 675-81.

17. Chicharro JL, Lucia A, Perez $M$, Vaquero AF, Urena R. Saliva composition and exercise. Sports Med 1998 Jul; 26(1):17-27.

18. Chen SD, Kao CH, Chang CS, Chen GH. Salivary function in patients with reflux esophagitis: effect of cisapride. J Nucl Med 1998 Aug; 39(8):1449-52.

19. Beal AM. The effect of transport-blocking drugs on secretion of fluid and electrolytes by the mandibular gland of red kangaroos Macropus rufus. Arch Oral Biol 1997 Oct-Nov; 42(10-11):70516.

20. Costa HO, Eckley CA, Fernandes AMF, Destailleur D, Villela PH. Refluxo gastroesofágico: comparação entre os achados laríngeos e digestivos. Rev Port ORL 1997; 35(1): 21-6.

21. Eckley CA \& Costa HO. Manifestações otorrinolaringológicas da doença do refluxo gastroesofágico In: Martinez JC. Atheneu Affecções Cirúrgicas do Estômago e Intestino Delgado. São Paulo; 2002 (no prelo)

22. Koufman JA. The otolaryngologic manifestations of gastroesophageal reflux disease (GERD): a clinical investigation of 225 patients using ambulatory 24-hour $\mathrm{pH}$ monitoring and an experimental investigation ofthe role of acid and pepsin in the development oflaryngeal injury. Laryngoscope 1991; 101(Suppl): 1-78.

23. Sarosiek J \& Mccallum RW. What Role do Salivary Inorganic Components Play in Health and Disease ofthe Esophageal Mucosa? Digestion 1995, 56 (Suppl 1): 24-31.

24. Sarosiek J \& Mccallum RW. Do Salivary Organic Components Play a Protective Role in Health and Disease of the Esophageal Mucosa? Digestion 1995; 56(Suppl 1): 32- 7

25. Shaw GY \& Searl JP. Laryngeal Manifestations of Gastroesophageal Reflux before and after Treatment with Omeprazole. S Med J 1997; 90(11): 1115-22.

26. Cianci R, Fedeli G, Cammarota G, Galli J, Agostino S, Di Girolamo S, Maurig M, Gasbarrini G. Is the alkaline reflux a risk factor for laryngeal lesions? Am J Gastroenterol 2000; 95(9): 2398 (carta). 